INVITED PARALLEL TALK

\section{Forward pion-nucleon charge exchange reaction and Regge constraints}

To cite this article: Huang Fei et al 2009 Chinese Phys. C 331318

View the article online for updates and enhancements.
Related content

- Gravity-matter entanglement in Regge
$\frac{\text { quantum gravity }}{\text { Nikola Paunkovi and Marko Vojinovi }}$
- IR divergences and Regge limits of
$\frac{\text { subleading-color contributions to the four- }}{\text { gluon amplitude in SYM theory }}$
Stephen G. Naculich and Howard J.
Schnitzer
- Sensitivity to the pion-nucleon coupling
$\frac{\text { constant in partial-wave analyses of N N, }}{\text { NN NN, and N N }}$
R A Arndt, I I Strakovsky, R L Workman et
al.

Recent citations

- Toward complete pion nucleon amplitudes V. Mathieu et al 


\title{
Forward pion-nucleon charge exchange reaction and Regge constraints*
}

\author{
HUANG Fei(黄飞) $)^{1 ; 1)}$ A. Sibirtsev ${ }^{2,3} \quad$ S. Krewald ${ }^{4,5} \quad$ C. Hanhart ${ }^{4,5}$ \\ J. Haidenbauer ${ }^{4,5}$ U.-G. Meißner ${ }^{2,4,5}$ \\ 1 (Department of Physics and Astronomy, University of Georgia, Athens, Georgia 30602, USA) \\ 2 (Helmholtz-Institut für Strahlen- und Kernphysik und Bethe Center for Theoretical Physics, \\ Universität Bonn, D-53115 Bonn, Germany) \\ 3 (Excited Baryon Analysis Center, Thomas Jefferson National Accelerator Facility, Newport News, Virginia 23606, USA) \\ 4 (Institut für Kernphysik and Jülich Center for Hadron Physics, Forschungszentrum Jülich, D-52425 Jülich, Germany) \\ 5 (Institute of Advanced Simulation, Forschungszentrum Jülich, D-52425 Jülich, Germany)
}

\begin{abstract}
We present our recent study of pion-nucleon charge exchange amplitudes above $2 \mathrm{GeV}$. We analyze the forward pion-nucleon charge exchange reaction data in a Regge model and compare the resulting amplitudes with those from the Karlsruhe-Helsinki and George-Washington-University partial-wave analyses. We explore possible high-energy constraints for theoretical baryon resonance analyses in the energy region above $2 \mathrm{GeV}$. Our results show that for the pion-nucleon charge exchange reaction, the appropriate energy region for matching meson-nucleon dynamics to diffractive scattering should be around $3 \mathrm{GeV}$ for the helicity flip amplitude.
\end{abstract}

Key words pion-nucleon interaction, Regge model

PACS 13.75.-n, 14.20.Gk, 11.55.Jy

\section{Introduction}

The study of baryon resonances is always an interesting topic, and recently there is intense experimental activity to study baryon resonances in the energy range up to $2.4 \mathrm{GeV}^{[1-4]}$. Theoretically, the study of baryon resonances in the high energy range requires an extension of the present theoretical meson-baryon dynamical coupled-channel models from low energy range to energies above $2 \mathrm{GeV}$. In principle, partial wave amplitudes would provide the most helpful tool for such a challenging task of extension. But, there are limitations at large energies, i.e. partial wave analyses may suffer from convergence problems. For example, in the energy range from $2 \mathrm{GeV}$ to $3.5 \mathrm{GeV}$, the number of partial waves employed in the KH80 analysis rises, as partial waves up to angular momentum $j=37 / 2$ are necessary. Naturally, it is difficult to determine such a large number of parameters from the available data.
For very high energies the angular distributions of two-body reactions are dominated by forward scattering. Here an economic description of the $t$ dependence of the cross sections in forward direction as well as of their energy dependence is given by Regge phenomenology ${ }^{[5]}$, using Regge trajectories as basic degrees of freedom. Therefore, the question arises in how far the amplitudes deduced from such approaches can serve as a guideline for the envisaged extension to higher energies.

In this talk, we report on a recent study of pionnucleon charge exchange amplitudes above $2 \mathrm{GeV}^{[6]}$. We survey the available information on the $\pi \mathrm{N}$ scattering amplitudes from $2 \mathrm{GeV}$ up to energies where the reaction can be quantitatively described within Regge phenomenology. Specifically, we consider the amplitudes that result from the partial wave analyses of the GWU group (which reaches up to $2.5 \mathrm{GeV}$ ) and the KH80 solution (which covers energies up to $3.49 \mathrm{GeV}$ ) and predictions of Regge models, fitted to

Received 7 August 2009

* Supported by Helmholtz Association through funds provided to the virtual institute "Spin and strong QCD" (VH-VI-231), EU Integrated Infrastructure Initiative Hadron Physics Project (RII3-CT-2004-506078), DFG (SFB/TR 16, "Subnuclear Structure of Matter"), JLab (SURA-06-C0452) and COSY FFE (41760632)

1) E-mail: huang@physast.uga.edu

(C)2009 Chinese Physical Society and the Institute of High Energy Physics of the Chinese Academy of Sciences and the Institute of Modern Physics of the Chinese Academy of Sciences and IOP Publishing Ltd 
high energy $\pi \mathrm{N}$ data.

In section 2, we briefly introduce the employed Regge model. The angular distributions and polarizations obtained are shown in section 3 for some selected energies, followed by a discussion of the energy and momentum dependence of the differential cross sections. The resulting amplitudes are presented and discussed in section 4 . Conclusions are drawn in section 5 .

\section{Formalism}

The helicity spin non-flip amplitude $\mathcal{M}_{\rho}^{++}$and spin-flip amplitude $\mathcal{M}_{\rho}^{+-}$due to the $\rho$-pole exchange contribution to the $\pi^{-} \mathrm{p} \rightarrow \pi^{0} \mathrm{n}$ reaction are parametrized as

$$
\begin{aligned}
& \mathcal{M}_{\rho}^{++}=\beta_{\rho}^{++} \frac{\pi G_{\rho}(s, t)}{\Gamma\left[\alpha_{\rho}(t)\right]}, \\
& \mathcal{M}_{\rho}^{+-}=\sqrt{-t} \beta_{\rho}^{+-} \frac{\pi G_{\rho}(s, t)}{\Gamma\left[\alpha_{\rho}(t)\right]},
\end{aligned}
$$

where $\beta_{\rho}$ is the residue function specified later. The Regge propagator is given by

$$
G_{\rho}(s, t)=\frac{1+\xi_{\rho} \exp \left[-\mathrm{i} \pi \alpha_{\rho}(t)\right]}{\sin \left[\pi \alpha_{\rho}(t)\right]}\left(\frac{s}{s_{0}}\right)^{\alpha_{\rho}(t)},
$$

with $\xi_{\rho}=-1$ being the signature of the $\rho$-trajectory. The $\rho$ trajectory $\alpha_{\rho}(t)$ is taken as

$$
\alpha_{\rho}(t)=1-\alpha_{\rho}^{\prime} m_{\rho}^{2}+\alpha_{\rho}^{\prime} t
$$

The slope parameter $\alpha_{\rho}^{\prime}$ is determined by a fit to the data. $s$ in Eq. (3) is the invariant collision energy squared and $s_{0}=1 \mathrm{GeV}^{2}$ serves as a scale. The $\Gamma$ function cancels the poles of the Regge propagator of Eq. (3) in the scattering region.

The spin non-flip amplitude $\mathcal{M}_{\mathrm{c}}^{++}$and spin flip amplitude $\mathcal{M}_{\mathrm{c}}^{+-}$due to the $\rho$-cut exchange, which represents the initial and final state interactions, are parametrized as

$$
\begin{aligned}
& \mathcal{M}_{\mathrm{c}}^{++}=\beta_{\mathrm{c}}^{++} \frac{\pi G_{\mathrm{c}}(t, s)}{\Gamma\left[\alpha_{\mathrm{c}}(t)\right]} \ln ^{-1}\left(s / s_{0}\right), \\
& \mathcal{M}_{\mathrm{c}}^{+-}=\sqrt{-t} \beta_{\mathrm{c}}^{+-} \frac{\pi G_{\mathrm{c}}(t, s)}{\Gamma\left[\alpha_{\mathrm{c}}(t)\right]} \ln ^{-1}\left(s / s_{0}\right),
\end{aligned}
$$

where the prescription of $G_{\mathrm{c}}(t, s)$ is similar to that of $G_{\rho}(t, s)$ except that $\alpha_{\rho}(t)$ is replaced by $\alpha_{\mathrm{c}}(t)$. Here $\alpha_{\mathrm{c}}(t)$ is the $\rho$-cut trajectory taken as

$$
\alpha_{\mathrm{c}}(t)=1-\alpha_{\rho}^{\prime} m_{\rho}^{2}+\frac{\alpha_{\rho}^{\prime} \alpha_{\mathrm{P}}^{\prime}}{\alpha_{\rho}^{\prime}+\alpha_{\mathrm{P}}^{\prime}} t
$$

with $\alpha_{\mathrm{P}}^{\prime}=0.1 \mathrm{GeV}^{-2}$ being the slope of the Pomeron trajectory, which is well defined from the analysis of elastic scattering data.
The residue functions for all amplitudes are parameterized in a similar way, namely by

$$
\beta(t)=\beta_{0} \exp (b t)
$$

where the coupling constant $\beta_{0}$ and the slope $b$ in the exponential formfactor are determined by a fit to the data.

The total helicity spin non-flip amplitude $\mathcal{M}^{++}$ and spin-flip amplitude $\mathcal{M}^{+-}$are given by the sum of the above amplitudes, i.e.

$$
\begin{aligned}
& \mathcal{M}^{++}=\mathcal{M}_{\rho}^{++}+\mathcal{M}_{\mathrm{c}}^{++}, \\
& \mathcal{M}^{+-}=\mathcal{M}_{\rho}^{+-}+\mathcal{M}_{\mathrm{c}}^{+-} .
\end{aligned}
$$

The differential cross section is given by

$$
\frac{\mathrm{d} \sigma}{\mathrm{d} t}=\frac{\left|\mathcal{M}^{++}\right|^{2}+\left|\mathcal{M}^{+-}\right|^{2}}{s q^{2}}
$$

and the polarization by

$$
P=\frac{2 \operatorname{Im}\left[\mathcal{M}^{++} \mathcal{M}^{+-*}\right]}{\left|\mathcal{M}^{++}\right|^{2}+\left|\mathcal{M}^{+-}\right|^{2}} .
$$

The difference of the $\pi^{-} \mathrm{p}$ and $\pi^{+} \mathrm{p}$ total cross sections is

$$
\Delta \sigma \equiv \sigma_{\pi^{-} \mathrm{p}}-\sigma_{\pi^{+} \mathrm{p}}=-\frac{4 \sqrt{2 \pi}}{q \sqrt{s}} \operatorname{Im} \mathcal{M}^{++}(t=0) .
$$

The parameters are listed in Table 1 . Those parameters have been determined by fitting the data on $\pi^{-} \mathrm{p} \rightarrow \pi^{0} \mathrm{n}$ angular distributions and polarizations for pion beam momenta above $4 \mathrm{GeV}(\sqrt{s} \geqslant 3 \mathrm{GeV})$ and for four-momentum transfer squared $|t| \leqslant 2 \mathrm{GeV}^{2}$.

Table 1. Parameters of the amplitudes.

\begin{tabular}{crr}
\hline Parameter & \multicolumn{1}{c}{$\rho$} & \multicolumn{1}{c}{$\rho$-cut } \\
\hline$\beta_{0}^{++} /\left[\mu \mathrm{b}^{1 / 2} \cdot \mathrm{GeV}\right]$ & $-23.8 \pm 0.3$ & $0.5 \pm 0.3$ \\
$b^{++} / \mathrm{GeV}^{-2}$ & $2.5 \pm 0.2$ & $0.6 \pm 0.4$ \\
$\beta_{0}^{+-} / \mu b^{1 / 2}$ & $151.3 \pm 1.1$ & $-113.7 \pm 2.2$ \\
$b^{+-} / \mathrm{GeV}^{-2}$ & $1.7 \pm 0.1$ & $4.3 \pm 0.3$ \\
\hline$\alpha_{\rho}^{\prime} / \mathrm{GeV}^{-2}$ & \multicolumn{2}{c}{$0.81 \pm 0.01$} \\
\hline
\end{tabular}

\section{Cross sections and polarization}

The differential cross sections and polarizations obtained from our Regge-cut model are shown in Figs. 1 and 2 for a few selected energies. A more systematic comparison with data will be presented elsewhere $^{[7]}$. In Figs. 1 and 2, the solid lines represent the results from our Regge-cut model. The data for the differential cross sections are taken from Refs. [8-14], and the data for the polarization are taken from Refs. [15-19].

From Fig. 1 one sees that our Regge-cut model reproduces the data on differential cross section reasonably well. In the Regge-cut model, the contribution 
of the rho-pole dominates both the helicity non-flip and the helicity flip amplitudes. The rho-cut amplitudes are a correction which are mainly required to fill the dip near $-t=0.6 \mathrm{GeV}^{2}$. One observes a maximum of the angular distribution near $-t=0.03 \mathrm{GeV}^{2}$ which allows to disentangle the helicity flip and nonflip amplitudes.

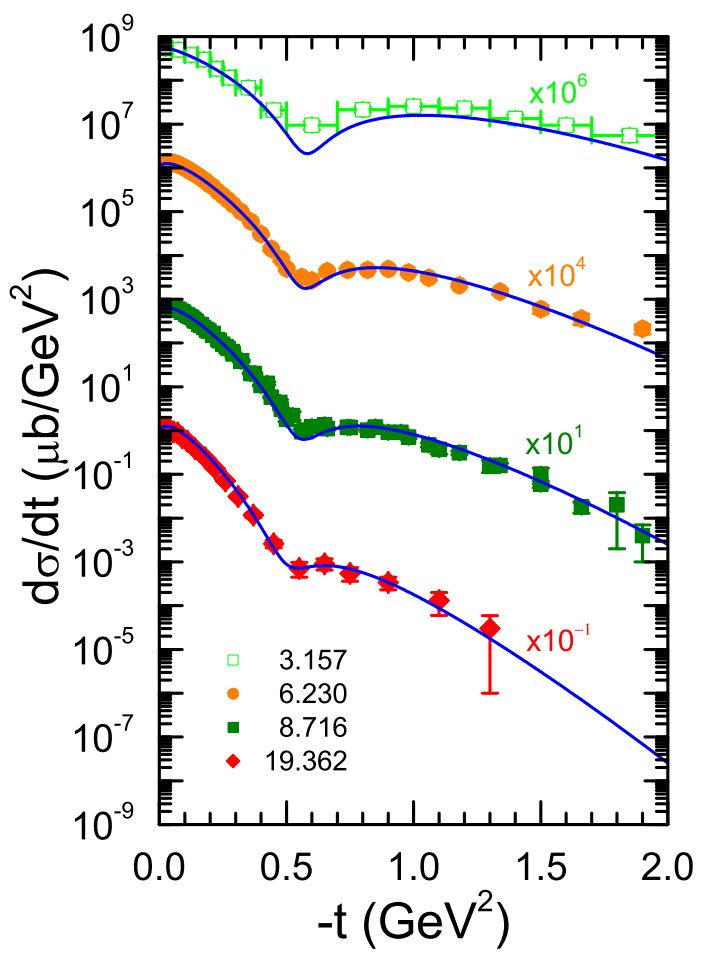

Fig. 1. Differential cross sections for the reaction $\pi^{-} \mathrm{p} \rightarrow \pi^{0} \mathrm{n}$ as a function of the fourmomentum transfer squared for different collision energies indicated in the legend.

As is well known, the pure Regge-pole model predicts zero polarization since there is no relative phase between the spin flip and spin non-flip amplitudes (see Eqs. (1) and (2)). With the rho-cut amplitudes included in our Regge model, we get results for the polarization very close to the data (see Fig. 2). Here the maximum of the polarization which occurs close to $-t=0.6 \mathrm{GeV}^{2}$, is correlated with the first minimum of the angular distributions, as expected from Eq. (12). One notes that the experimental data have large uncertainties, and different sets even conflict. To get a better theoretical description of the $\pi^{-} \mathrm{p} \rightarrow \pi^{0} \mathrm{n}$ reaction, more precise polarization data are needed.

The helicity flip and non-flip cross sections are observables which, in principle, could be measured directly. The differential cross section for $t=0$ is entirely determined by the helicity non-flip transition, which opens the possibility to separate the two

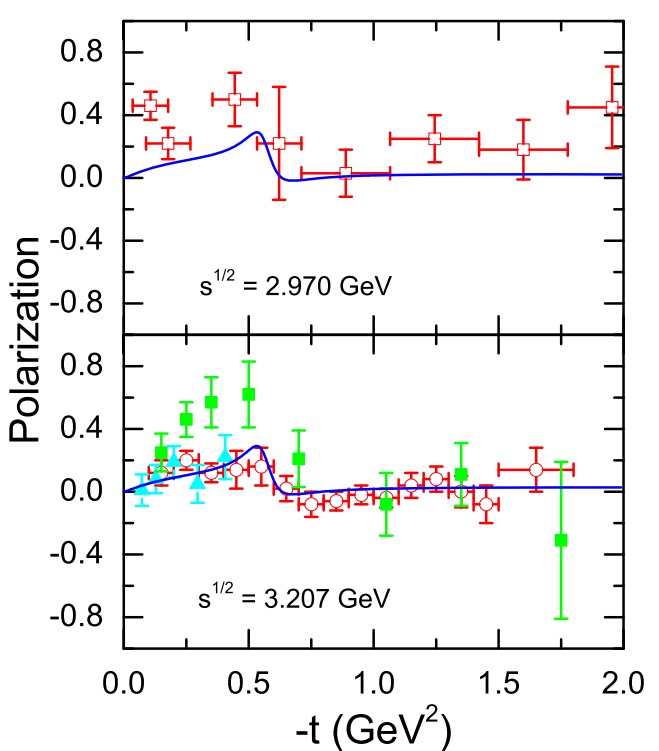

Fig. 2. The polarization in the reaction $\pi^{-} \mathrm{p} \rightarrow$ $\pi^{0} \mathrm{n}$ as a function of four-momentum transfer squared for different collision energies indicated in the legends.

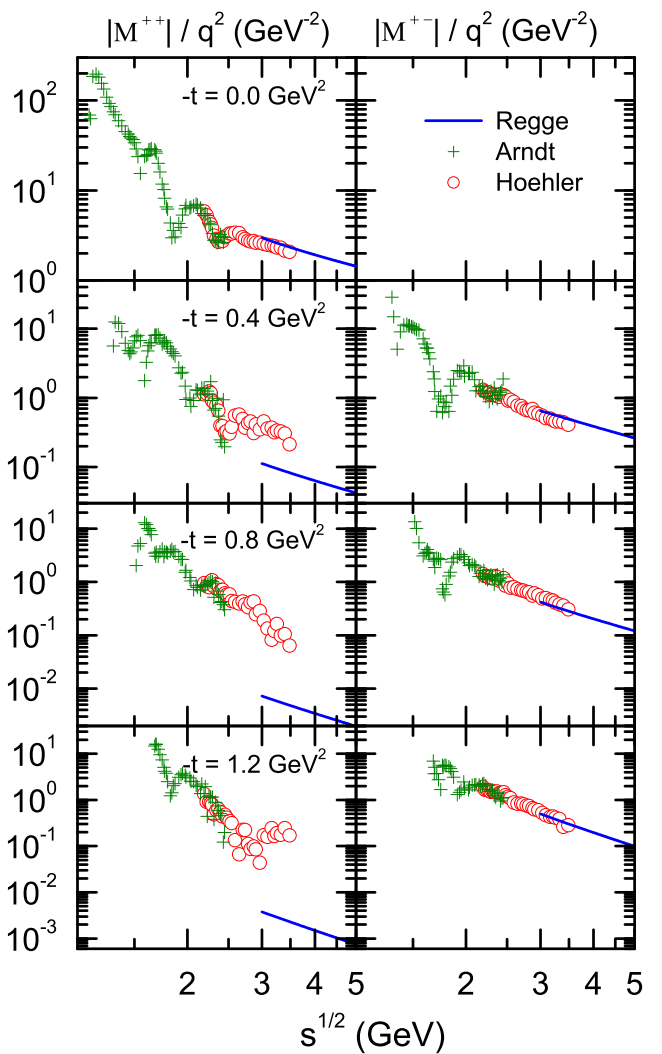

Fig. 3. Moduli of the spin non-flip and spinflip amplitudes for the reaction $\pi^{-} \mathrm{p} \rightarrow \pi^{0} \mathrm{n}$ divided by the center-of-mass momentum squared at fixed $t$ as a function of the collision energy $\sqrt{s}$. The solid lines represent the results from our Regge model. The "+" symbols and the open circles represent the results of the partial wave analysis from the GWU group and the Karlsruhe-Helsinki group, respectively. 
contributions to the cross section within the Regge model by studying the $t$-dependence of the cross sections. In Fig. 3, we show the energy dependence of the moduli of the helicity flip and non-flip amplitudes. Here we also include results based on two $\pi \mathrm{N}$ phase shift analyses, namely the ones by the GWU ${ }^{[20]}$ and the Karlsruhe-Helsinki ${ }^{[21]}$ groups. The results for the GWU analysis are those of their current solution taken from the SAID Program ${ }^{[22]}$. With regard to Karlsruhe-Helsinki we use the preliminary updated solution KH80 as tabulated in Table 2.2.2.2 of Ref. [21].

For the helicity flip amplitude, one sees that the KH80 analysis joins the Regge fits smoothly. For the helicity non-flip amplitude, the moduli match at $t=0$, as expected, but at larger four-momentum transfers, the Regge-cut model produces an amplitude which is much smaller than the KH80 result. The helicity nonflip amplitudes of the KH80 analysis show large fluctuations above $2.5 \mathrm{GeV}$ for all values $-t \geqslant 0.8 \mathrm{GeV}^{2}$. One should note that the helicity non-flip amplitude is fairly small above $\sqrt{s} \approx 2.5 \mathrm{GeV}$, which makes its determination difficult. Direct measurements of polarization and spin rotation parameters for forward angles would help.

\section{Amplitudes}

In Figs. 4 and 5 we present a systematic comparison of the energy dependence of the amplitudes from our Regge model and those from partial wave analyses by the $\mathrm{GWU}^{[20]}$ and the Karlsruhe-Helsinki ${ }^{[21]}$ groups.

Let us first discuss the real and imaginary parts of the helicity flip amplitudes which are shown in Fig. 4. For energies from around $\sqrt{s} \approx 2.3 \mathrm{GeV}$ onwards the GWU analysis and the KH80 analysis start to deviate from each other. For energies above $\sqrt{s} \approx 3 \mathrm{GeV}$ the imaginary parts of the amplitudes from KH80 are close to those from our Regge model for practically all $t$-values considered. The real parts of the helicity flip amplitude generated by our Regge fit are not in line with the KH80 results. The largest difference occurs around $-t \approx 0.8 \mathrm{GeV}^{2}$.

The amplitudes for the helicity non-flip amplitudes are shown in Fig. 5. Also here one observes a deviation of the GWU amplitudes from the KH80 result from around $2.3 \mathrm{GeV}$ onwards. For $t=0$, the KH80 analysis and our Regge model are in reasonable agreement for energies above $\sqrt{s} \approx 2.5 \mathrm{GeV}$. Note that $\operatorname{Im} M^{++}(t=0)$ is proportional to the difference of the $\pi^{-} \mathrm{p}$ and $\pi^{+} \mathrm{p}$ cross section (see Eq. (13)) and,

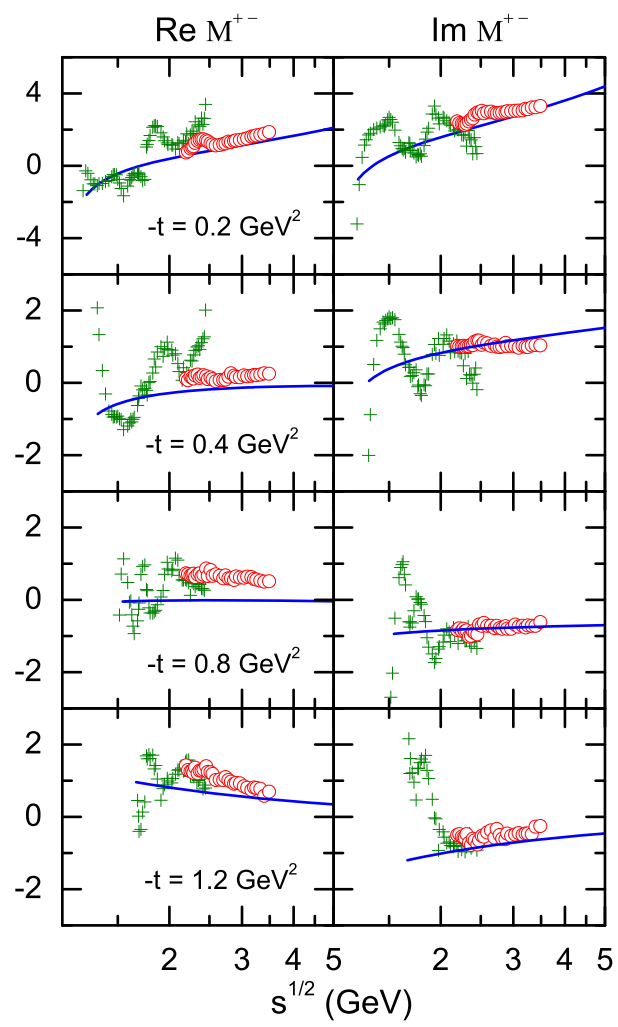

Fig. 4. Helicity flip amplitudes for the reaction $\pi^{-} \mathrm{p} \rightarrow \pi^{0} \mathrm{n}$ at fixed $t$ as a function of the collision energy $\sqrt{s}$. Same description of curves as in Fig. 3.

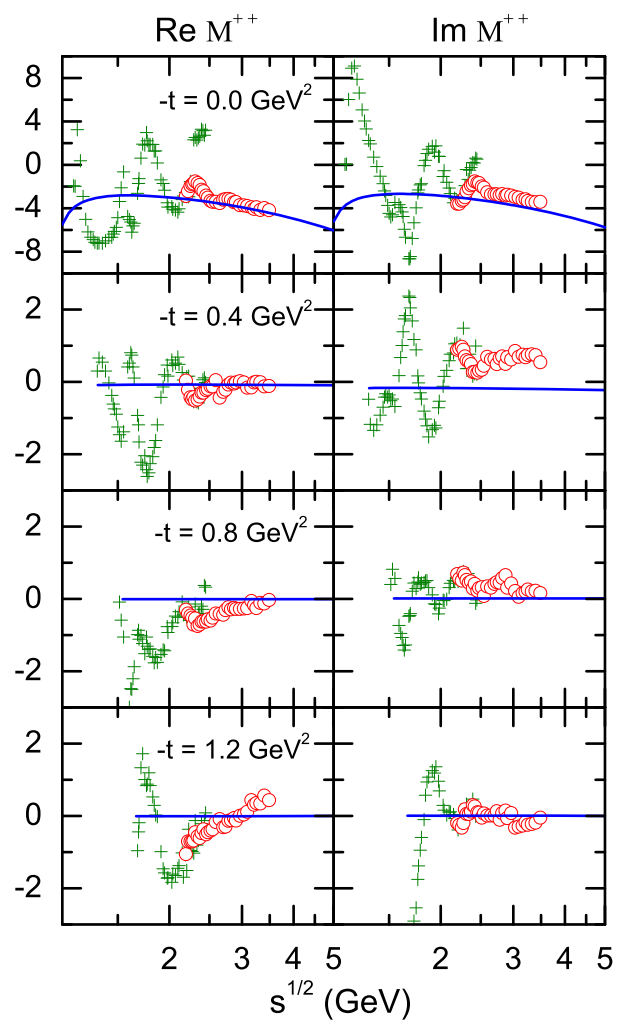

Fig. 5. Helicity non-flip amplitudes for the reaction $\pi^{-} \mathrm{p} \rightarrow \pi^{0} \mathrm{n}$ at fixed $t$ as a function of the collision energy $\sqrt{s}$. Same description of curves as in Fig. 3. 
therefore, an empirically accessible quantity. For $-t \geqslant 0.8 \mathrm{GeV}^{2}$, the result for $\operatorname{Im} M^{++}$from our Reggecut model approaches zero as suggested by the Karlsruhe Helsinki analysis. Surprisingly, the real part of $M^{++}$of the KH80 analysis exhibits a significant energy dependence for fairly large values of $|t|$ which is not reproduced by the Regge-cut model.

\section{Conclusions}

We have presented a Regge-cut model which reproduces the differential cross sections down to energies $\sqrt{s} \approx 3 \mathrm{GeV}$ and for four-momentum transfer squared $-t<2 \mathrm{GeV}^{2}$. We then compared the resulting amplitudes with those determined in the
Karlsruhe-Helsinki (KH80) partial wave analysis. It turned out that the magnitudes of the helicity non-flip amplitudes are not well constrained, while the magnitudes of the helicity flip amplitudes derived from the Regge-cut model join the corresponding quantities obtained in the KH80 partial wave analysis smoothly in the vicinity of $\sqrt{s}=3 \mathrm{GeV}$. We conclude that the appropriate energy region for matching mesonnucleon dynamics to diffractive scattering could be around approximately $3 \mathrm{GeV}$ for the helicity flip amplitude of the $\pi \mathrm{N}$ charge-exchange reaction.

F.H. is grateful for the support from the Alexander von Humboldt Foundation during his stay in Forschungszentrum Jülich where the main part of this work was completed.

\section{References}

Sarantsev A V et al. Phys. Lett. B, 2008, 659: 94-100

Aznauryan I G et al. Phys. Rev. C, 2008, 78: 045209

3 Nasseripour R et al. Phys. Rev. C, 2008, 77: 065208

4 Sumihama M et al. Phys. Lett. B, 2007, 657: 32-37

5 Donnachie A, Landshoff P V. Phys. Lett. B, 1992, 296: $227-232$

6 Huang F, Sibirtsev A, Krewald S, Hanhart C, Haidenbauer J, Meißner U G. Eur. Phys. J. A, 2009, 40: 77-87

7 Sibirtsev $\mathrm{A}$ et al. In preparation.

8 Apel W D et al. Phys. Lett. B, 1977, 72: 132

9 Apokin V D et al. Z. Phys. C, 1982, 15: 293

10 Barnes A V et al. Phys. Rev. Lett., 1976, 37: 76

11 Bolotov V N et al. Nucl. Phys. B, 1974, 73: 365-386
12 Guisan O, Bonamy P, Du P L, Paul L. Nucl. Phys. B, 1971, 32: $681-690$

13 Sonderegger $\mathrm{P}$ et al. Phys. Lett., 1966, 20: 75

14 Wahlig M A, Mannelli I. Phys. Rev., 1968, 168: 1515

15 Minowa M et al. Nucl. Phys. B, 1987, 294: 979-1000

16 Bonamy $\mathrm{P}$ et al. Nucl. Phys. B, 1973, 52: 392-402

17 Hill D et al. Phys. Rev. Lett., 1973, 30: 239-242

18 Drobnis D D et al. Phys. Rev. Lett., 1968, 20: 274

19 Giacomelli G. Landolt-Börnstein Series, Group I, Elementary Particles, Nuclei and Atoms 7. Berlin: Springer, 1973

20 Arndt R A, Briscoe W J, Strakovsky I I, Workman R L. Phys. Rev. C, 2006, 74: 045205

21 Höhler G. Landolt-Börnstein Series, Group I, Elementary Particles, Nuclei and Atoms 9b1. Berlin: Springer, 1983.

22 Arndt R A, Briscoe W J, Workman R L, Strakovsky I I. CNS Data Analysis Center, http://gwdac.phys.gwu.edu/ 